\title{
Detecting anthropogenic effects in the observational evidence of climate change
}

\section{H. von Storch}

Director of the Institute for Coastal Research, GKSS Research Center, Geesthacht, Germany. Professor at the Meteorological Institute of the University of Hamburg, Germany. Email: hvonstorch@web.de

Manuscript received: September 2008; accepted: September 2008

\begin{abstract}
The issue of detecting changes beyond the range of natural variability (detection) and of attributing causes to such changes (attribution) are central to any rational debate about anthropogenic climate change. This concept, introduced by Klaus Hasselmann in the late 1970s, is usually not understood by either so-called sceptics or by activist scientists.

often rigorous detection and attribution analysis is replaced by mere declarations and by naïve applications of methods to determine if certain trends are 'significant' or not.

In this talk, the concepts are introduced; the invoked assumptions and the roles of dynamical models and of time-scales are explained. The concept is illustrated with a few examples related to global and regional air temperature and to NE Atlantic storminess.

For global and regional air temperature, the recent variations are found to be beyond the range of natural variability (detection) and the changes are best explained by a dominant contribution by elevated greenhouse gas concentrations. For NE Atlantic storminess recent trends are found to be intermittent and within the historical range of variations. The finding of significant GHG contributions to temperature change and the lack of such for changes in storminess are not contradictions.
\end{abstract}

